\title{
CREATING DIGITAL ELEVATION MODEL USING A MOBILE DEVICE
}

\author{
A.İ. DURMAZ a \\ ${ }^{a}$ NCSU, Geospatial Information Science \& Tech., Raleigh NC, US- aliihsandurmaz@ gmail.com
}

KEY WORDS: Dem, Digital Elevation Model, Mobile Device, GPS

\begin{abstract}
:
DEM (Digital Elevation Models) is the best way to interpret topography on the ground. In recent years, lidar technology allows to create more accurate elevation models. However, the problem is this technology is not common all over the world. Also if Lidar data are not provided by government agencies freely, people have to pay lots of money to reach these point clouds. In this article, we will discuss how we can create digital elevation model from less accurate mobile devices' GPS data. Moreover, we will evaluate these data on the same mobile device which we collected data to reduce cost of this modeling.
\end{abstract}

\section{INTRODUCTION}

Obtaining terrain parameters, extracting flow lines, erosion modeling, structural engineering design, and surface analysis etc. These are some common DEM (Digital elevation model) application areas. To create a digital elevation model we need both ground and elevation coordinates for same location. These coordinate data can be gathered by lidar, RTK (Real time kinematic - GPS method) or total station. After ground and elevation coordinate data obtained, evaluation progress starts and in this progress we have to deal with too many coordinates so we have to use electronic progressers, computers, in this step.

Nowadays, lots of new technological devices like tablet pcs, smart phones or navigation systems have their own operation systems with interior GPS drivers. These smart devices can measure ground and elevation coordinates using momentary satellite signals with their interior GPS drivers. In addition, some of these devices can be captured GPS coordinates in .kml, or .gpx file type by using suitable applications. We have to say that this positioning system is not accurate like RTK GPS method but these data's accuracy is enough for these devices' aims (especially navigation). In this article, we will create an elevation model using these smart phones' coordinate data and we will evaluate these data on their own operation systems. Moreover, we will compare our result maps with bare earth lidar data elevation model to check accuracy of created elevation model.

We picked out Nokia N900 smart phone as our working device because its Linux base operation system allows to install open source GIS programs like Grass or QGIS. In this project we preferred to work with Grass rather than QGIS on our mobile device because firstly Grass performance is better than QGIS and Grass has powerful algorithms to create surfaces from point data.

\section{MATERIALS}

\subsection{Study Site}

The 7.5-ha study site is located in Raleigh, NC. (Figure 1). The study site is a free agriculture area it does not contain artificial object which can block satellite signals. According to bare earth lidar data there is about 15 meter elevation difference between maximum and minimum elevation points in this site. The geographic coordinate of study site is $\left(35^{\circ} .73647 \mathrm{~N}\right.$, $\left.078^{\circ} .68056 \mathrm{~W}\right)$.

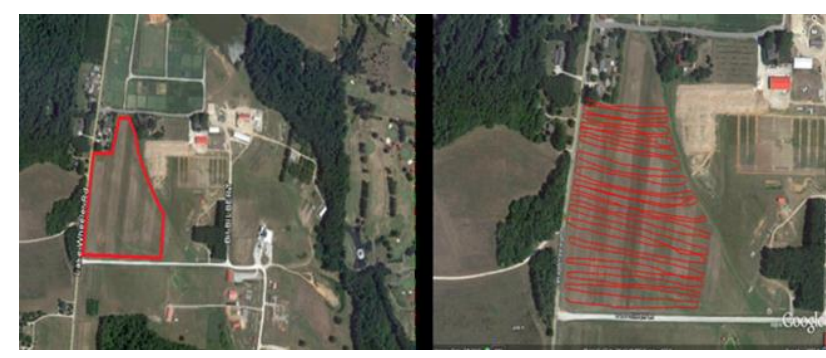

Figure 1. Figure placement and numbering

\subsection{Data}

Bare earth lidar data of our study site is provided by North Carolina Foodplain Mapping program(www.ncfloodmaps.com). The horizontal coordinate system of this point cloud is NAD 1983 State Plane North Carolina and its unit is in feet scale. The vertical coordinate system of point cloud is NAVD88 (North American Vertical Datum 88) and its unit is again in feet scale.

GPS data in study site are gathered by Nokia N900's GPS deriver. GPS data's horizontal and vertical coordinate systems are WGS84. Its horizontal coordinate units are in decimal values of latitude and longitude and the vertical unit is in meter scale.

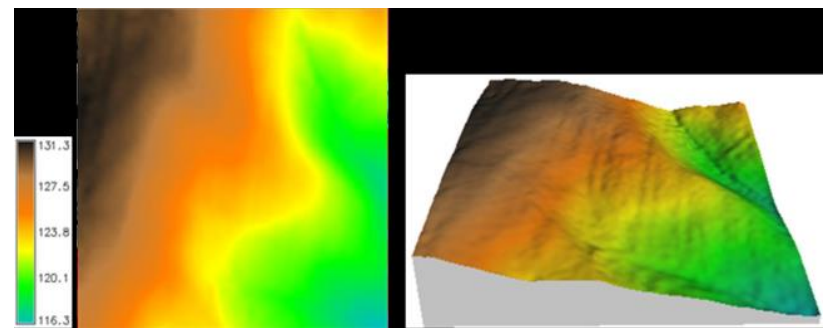

Figure 2. Bare earth elevation model under normal tension without smoothing parameter

Lidar data used to create DEM map of study site using spline method under normal tension and without smoothing parameter (Figure 2) in $1 \times 1$ meter resolution. We assume this elevation model is our site's certain elevation model so we convert GPS data's horizontal coordinate to bare earth lidar data's coordinate system and unit to compare our elevation model with bare earth lidar data elevation model. 


\subsection{Comparing Lidar Data with GPS Data Which Obtained from Mobile Device}

In study site there are 6222 GPS points and 3660 lidar points. Although GPS data have more points, it does not completely cover study site like lidar data. GPS data points are only dense on walking route. There are about 10 to 15 meter gaps between two walking line in GPS data. On the other hand, this gap distance between two points is lower than 3 meter in bare earth lidar data (Figure 3).

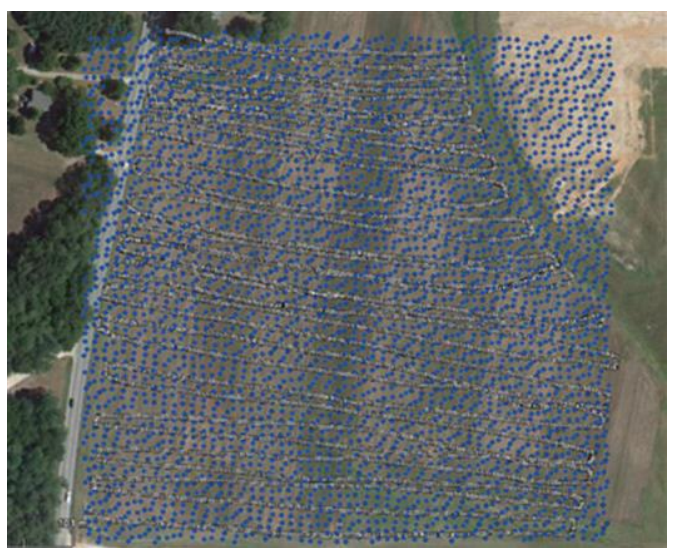

Figure 3. Bare earth lidar data and GPS data on project site

The other point between these point data sets is that GPS data are less sensitive than bare earth lidar data. Mobile device's GPS driver can measure only half meter elevation differences but lidar data can measure 1 centimeter elevation difference on the ground. That means Lidar data are 500 times sensitive than GPS elevation points.

In addition, if we compare accuracy of GPS point clouds with bare earth lidar data we will see that there are big elevation jumps between two close points. For instance, while we were collecting GPS data sometimes our route lines were crossing. In one of these crossing lines, there is 17 meter vertical difference in 21 centimeter horizontal distance on the ground (Figure 4). (One of these lines eliminate before creating elevation model because of preventing ground shape deformation otherwise one of these lines will create a big hill and the other one will create a deep hole in a close area)

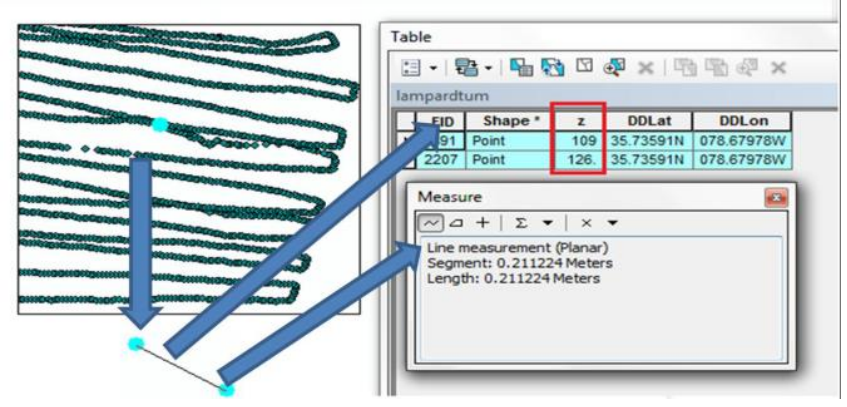

Figure 4. Accuracy of GPS data

\section{METHODS AND APPROACHES}

Voronoi, IDW (inverse distance weight), and radial basis functions and spline methods are available on GRASS to create digital elevation model from point data.

\subsection{Voronoi}

"Voronoi method is that the test area was divided into subareas by finding the centers of mass of the source data point groups and calculating Voronoi regions based on these centers. Dividing an area using the Voronoi algorithm is an important tool in computational geometry. It is based on the principle that all the points closer to a particular center point than to any other center points belong to the same Voronoi region. As a result, one Voronoi region for each center point was obtained. The borders between the computational packets are clearly visible introducing abrupt changes in the elevation values and the surface looks unnatural." (Pohjola, J., Turunen, J., Turunen, T. 2009)

Also, according to the Svec and Burden from university of Washington about problems of voronoi algorithm "We do not explicitly define simple, we loosely evaluate simplicity based on overall contiguity, compactness, convexity, and intuitiveness of the model's districts.” (Svec, L., Burden, S., Dilley, A. 2007)

Therefore, we abandoned using voronoi algorithm in our project site because discontinuity between polygons will create elevation jumps. Moreover, if we applied this method to our GPS points, lots of elevation polygons would have big edge differences because of distribution of GPS points.

\subsection{IDW (Inverse Distance Weighted Interpolation)}

"This interpolation method estimates a point using the nearest sample points, which are weighted by a power proportional to the inverse of their distance from the estimated point. The higher the power the stronger the influence of the closer sample points.” (Kurtzman, D., Kadmon, R., 1999)

The general equation of IDW interpolation method is;"

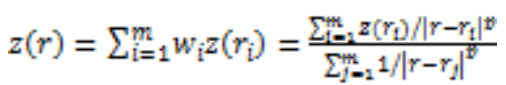

Where $\mathrm{p}$ is a parameter (typically $\mathrm{p}=2$, lower $\mathrm{p}$ gives smoother surface - similar to lower tension), and $\left|r-r_{i}\right|^{2}=\left(x-x_{i}\right)^{2}+\left(y-y_{i}\right)^{2}$ is the distance between the unsampled location $r$ and a given point ri. Smoothing can be introduced by adding a parameter $\beta$ to the weight term $\left|r-r_{i}\right|^{2}+\beta$, leading to approximation function.

Note: GRASS modules use $\mathrm{p}=2$ and $\mathrm{m}=12$ as default values." (Mitasova, H., Mitas, L. 1995)

The advantage of this method, it bases on computing average of elevation points so this can help to reduce the dense GPS point lines side effects.

3 different elevation maps created using IDW interpolation method (Figure 5) for 1 meter resolution from mobile device's GPS points. 12 (left image), 50 (middle image), and 200 (right image) are closest point counts which affected the weighted average respectively.

The disadvantages of this method computed elevation models are visually different then bare earth lidar data elevation model. GPS point lines create ridges, and gaps create valleys. If we compare these result maps with bare earth lidar data elevation model using map algebra method, we will see that approximately $80 \%$ of sites have less than 5 meter elevation mistake. (Table-1) 

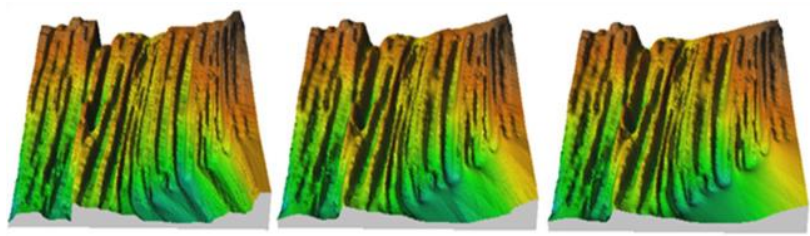

Figure 5. IDW method results

\begin{tabular}{|c|c|c|c|}
\hline \multicolumn{4}{|c|}{$\%$ differences with bare earth lidar data and IDW methods } \\
\hline & 5 meter & 3 meter & 1 meter \\
\hline 12 points & $80.74 \%$ & $58.29 \%$ & $23.33 \%$ \\
\hline 50 points & $82.74 \%$ & $60.31 \%$ & $25.17 \%$ \\
\hline 200 points & $83.45 \%$ & $62.53 \%$ & $26.18 \%$ \\
\hline
\end{tabular}

Table-1 IDW method \% correctly modeled places. According to different threshold values and point number

\subsection{Radial Basis Functions and Spline}

"Spline methods are based on the assumption that the approximation function should pass as closely as possible to the data points and should be at the same time as smooth as possible. These two requirements can be combined into a single condition of minimizing the sum of the deviations from the measured points and the smoothness seminorm of the function" (Mitasova, H., Mitas, L. 1995). The equation of spline modeling;

$$
\sum_{j=1}^{\mathbb{N}}\left|z_{j}-F\left(r_{j}\right)\right|^{2} w_{j}+w_{0} I(F)=\text { minimum }
$$

$W_{j}$, and $W_{0}$ are positive weights. $I(F)$ is smoothness seminorm

$$
F_{\gamma}=T_{(r)}+\sum_{j=1}^{N} \lambda_{j} R\left(r_{v} r_{j}\right)
$$

$T_{(r)}$ is trned function and $R\left(r_{v} r_{j}\right)$ is smoothness seminorm's function

$$
F_{(r)}=\alpha_{0}+\sum_{j=1}^{M} \lambda_{j}\left[K_{0}\left(\frac{\varphi r}{2}\right)+\ln \left(\frac{\varphi r}{2}\right)+C\right]
$$

In this equation $\varphi$ is tension, $\mathrm{C}$ is contant, $K_{0}$ is bessel and $\mathrm{r}$ is smoothness seminorm's function parameter

$$
F_{(r)}=\alpha_{0}-\sum_{j=1}^{N} \lambda_{j}\left[E_{1}(\varphi)+\ln (\varrho)+C_{E}\right]
$$

$C_{E}=0.577215$ is Euler constant. $E_{1}(Q)$ is exponential integral function and (e) is a tension function. Equation (6) and (7) can use to calculate $\alpha_{1}$ and $\lambda_{j}$.

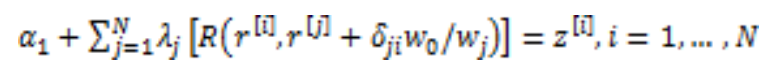

(6)

$$
\sum_{j=1}^{N} \lambda_{j}=0
$$

"The generalized tension $\varphi$ controls the distance over which the given point influences the resulting surface or hypersurface. For the bivariate case tuning the tension can be interpreted as tuning the character of the resulting surface between membrane and thin plate. The proper choice of smoothing and tension parameters is important for successful interpolation or approximation.” (Mitasova, H., Mitas, L. 1995).

Measured GPS points, as mentioned before, accuracy and sensitivity are too low so we need to use lower tension parameter and higher smoothing parameter to get good results. Default tension parameter is 40(Neteler, M. and Mitasova, H., 2008) and default smoothing parameter is 0

(Neteler, M. and Mitasova, H., 2008) in Grass. These parameters are in normal scale to create an elevation model from GPS or Lidar point clouds so we need to use lower value than 40 for tension parameter and higher value than 0 for smoothing parameter to create elevation model from GPS data. Figure 6 shows tension and smoothing parameters effect on elevation model.
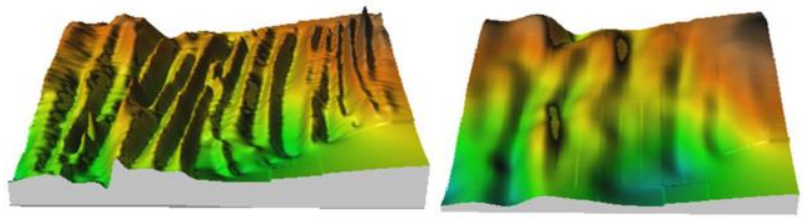

Figure 6. Tension and Smoothing parameters effect on elevation model left image 40 tension and 0 smoothing right image 10 tension and 10 smoothing

Furthermore, if we again compute elevation differences of these created models with bare earth lidar data elevation model, we will see that lower tension parameter and higher smoothing parameter model is more close to bare earth lidar data elevation model (Figure 7). However, tension and smoothing parameters are not enough to eliminate all artificial valleys and ridges. (Figure 6)

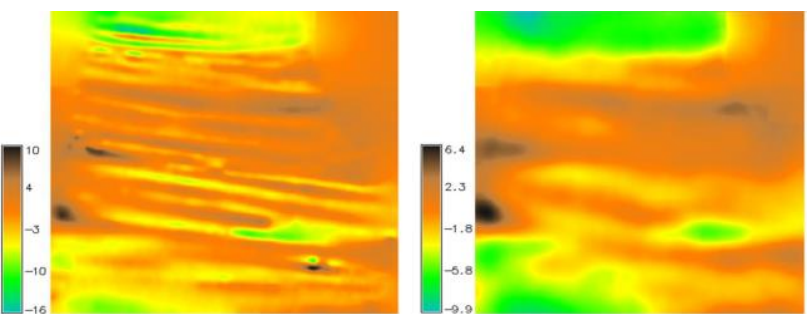

Figure 7. left image is elevation difference between bare earth lidar data and tension 40 smoothing 0 elevation model. Right image is elevation difference between bare earth lidar data and tension 10 smoothing 10 elevation model.

To eliminate these artificial valleys and ridges we need to define $r^{[0]} r^{[j]}$ parameter again in equation (6).

$$
r_{1}^{s}=r_{i} \cos (\theta)+r_{i} \sin (\theta)
$$

$r_{2}^{b}=-r_{i} \sin (\theta)+r_{i} \cos (\theta)$

In equation 8 and $9,(\theta)$ angle is named anisotropy angle. "Using the fact, that for the regularized spline with tension function, the change of scale is equivalent to the change in the tension parameter, anisotropy can be implemented by rotating the coordinate system by an angle ( $\theta$ ) (direction of anisotropy)" ( Hofierka, J., Parajka, J., Mitasova, H., Mitas, L. 2002). Then if we rescale $r_{1}$ axis according to anisotropy magnitude ("s" is anisotropy scale factor in equation 10.);

$$
v^{2}=s\left(r 1-r_{1}^{[j]}\right)^{2}+\left(r 1-r_{1}^{[j]}\right)^{2}
$$

We can eliminate artificial valleys and ridges in our elevation model. (Figure 8) 

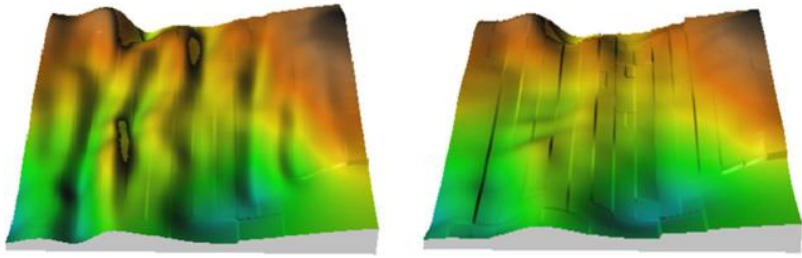

Figure 8. GPS data elevation models. Left image is tension 40 smoothing 0 and without anisotropy angle and scale factor.

Right image is tension 40 smoothing 0,25 degree anisotropy angle and 0.25 scale factor.

As a result, to create a better elevation model from gathered data we need to use lower tension, higher smoothing, anisotropy angle and anisotropy scale factor. Table- 2 shows what the percentage of modeled correctly area using $5 \mathrm{~m}, 3 \mathrm{~m}$ and $1 \mathrm{~m}$ threshold values (Table values are calculated using each model difference with bare earth lidar data elevation model).

\begin{tabular}{|l|l|l|l|}
\hline \multicolumn{4}{|c|}{ Spline Method Result Table } \\
\hline & 5 meter & 3 meter & 1 meter \\
\hline Tension 40, Smoothing 0 & $83.48 \%$ & $65.16 \%$ & $26.16 \%$ \\
\hline Tension 10, Smoothing 10 & $87.03 \%$ & $72.92 \%$ & $29.86 \%$ \\
\hline $\begin{array}{l}\text { Tension 10, Smoothing 10, } \\
\text { Anis. Angle 275 and Anis. } \\
\text { Scale factor 0.25 }\end{array}$ & $88.38 \%$ & $75.38 \%$ & $30.43 \%$ \\
\hline
\end{tabular}

Table-2 Spline method \% correctly modeled places base on different threshold values.

\subsection{Our Suggested Method}

Our previous experiments show that we need to use spline method (with lower tension, higher smoothing, and anisotropy angle and scale factor) rather than IDW method to create a better surface from gathered GPS data on GRASS. Therefore we used 0.1 tension parameter, 20 smoothing parameter 275 anisotropy angle and 0.25 anisotropy scale factor. However, lower tension and higher smoothing parameters create a surface which has big pixel sizes and elevation model does not look like continuous data. (Figure 9)

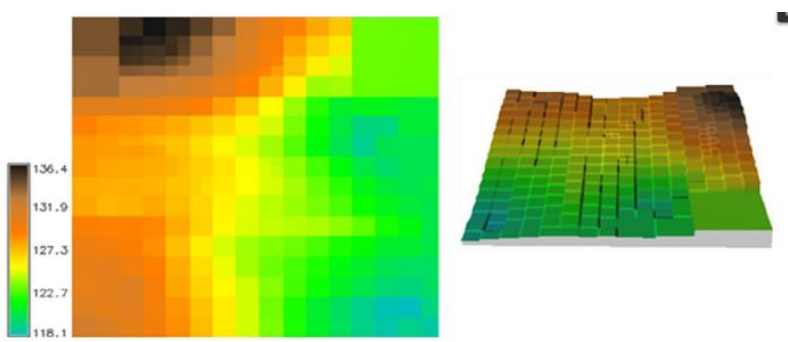

Figure 9. 0.1 tension parameter, 20 smoothing parameter 275 anisotropy angle and 0.25 anisotropy scale factor

To eliminate this discrete view and keep surface topologic shape we converted each pixel's centroid to point data using raster to point conversion tool in Grass. And then we created a new elevation model from new data points using spline method (40 tension, 0 smoothing and without anisotropy angle and scale factor) (Figure10 and Figure11).

If we compare last elevation model with bare earth lidar elevation model we will see that $95.71 \%$ area has less than 5 meter mistake, $80.46 \%$ area has less than 3 meter mistake and $30.45 \%$ area has less than 1 meter mistake.
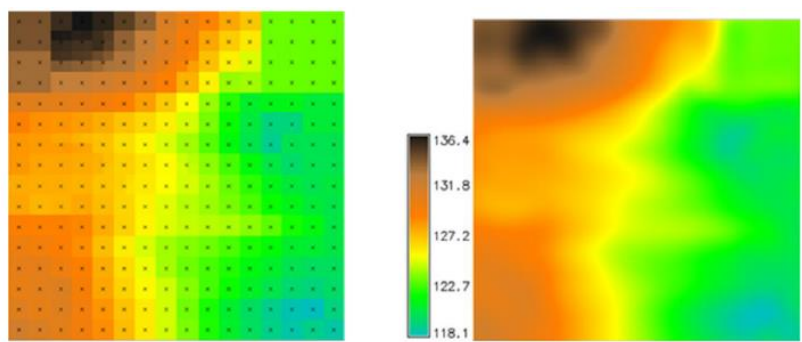

Figure 10. Left image shows new data points which were created by using raster to point conversion tool on Grass. Right image shows new elevation model which is created by using new data points.

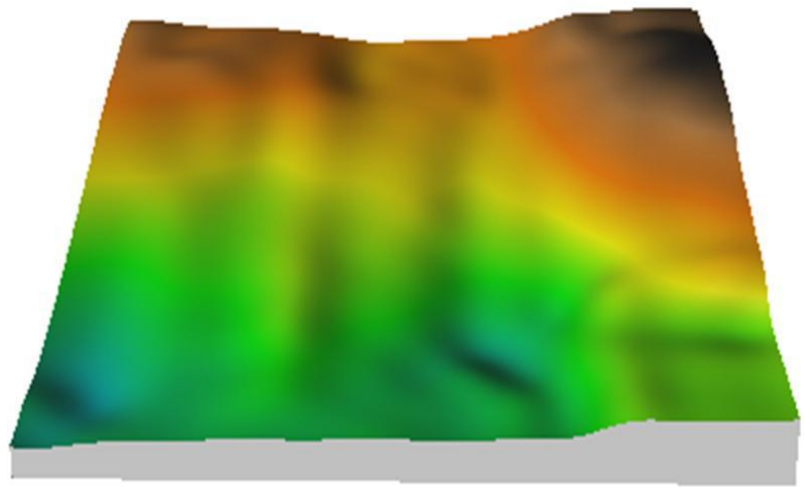

Figure11. 3D view of new elevation model

\section{CONCLUSION}

Mobile elevation model is not enough accurate extracting flow lines and erosion modeling but may be its accuracy will be enough for some engineering applications. The main reasons for incorrect modeling are that; firstly GPS data's low accuracy and sensitivity. Secondly, GPS data's vertical datum is different than lidar data vertical datum and lastly these two data did not gather simultaneous so may be surface would change between these two data gathering time. On the other hand the good side of this modeling its total cost less than 250 dollar.

This image bellow shows a digital elevation model directly captured from mobile device's screen (Figure 12). This elevation model was created by using our suggested method and computer did not use any part of this model.

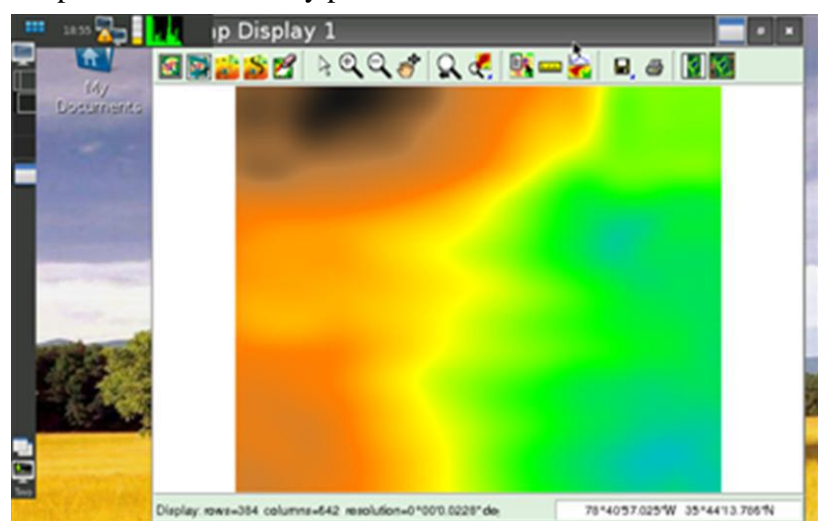

Figure 12. Elevation model in mobile device 


\section{REFERENCES}

Hofierka, J., Parajka, J., Mitasova, H., Mitas, L. 2002 Multivariate Interpolation of Precipitation Using Regularized Spline with Tension. Transactions in GIS, 2002, 6(2): 135 \pm 150

Kurtzman, D., Kadmon, R., 1999 Mapping of temperature variables in Israel: a comparison of different interpolation methods. Clim Res : 13 - 33

Mitasova, H., Mitas, L. 1995 Modeling spatially and temporally distributed phenomena: New methods and tools for GRASS GIS. Invited paper for the special issues of the international Journal of GIS 1995:1-23

Neteler, M. and Mitasova, H., 2008, Open Source GIS: A GRASS GIS Approach, 3rd Edition, Springer, New York, p 237

Pohjola, J., Turunen, J., Turunen, T. 2009 Creating HighResolution Digital Elevation Model Using Thin Plate Spline Interpolation and Monte Carlo Simulation. Posiva Working Report 2009-56:1 - 56

Svec, L., Burden, S., Dilley, A. 2007 Applying Voronoi Diagrams to the Redistricting Problem. The UMAP Journal 28.3 (2007) :313 - 330

\section{APPENDIX}

\section{WORKFLOWS}

These workflow steps show command lines for creating elevation model from gathered GPS data on mobile device

Import GPS data to mobile GRASS; (...) $\mathrm{kml}$ file location on mobile device

v.in.ogr -z dsn=(....)\project22.kml output=Points_GPS

Export .kml data to txt file. (this step is required because Grass does not read klm file z coordinate)

v.out.ascii input=Points_GPS output=......points.txt

Import GPS data points with $\mathrm{z}$ attribute column

v.in.ascii $-z$ input $=\ldots \ldots$... points.txt output=elevpoints $z=3$

Setting regions for 1 meter resolution (rows $=269$ cols=275 are equal 1 meter resolution values for my project site)

g.region vect $=$ elevpoints rows $=269$ cols $=275$

First, elevation model with lower tension, higher smoothing, anisotropy angle and scale.

v.surf.rst input=elevpoints elev=elev_GPS zcolumn=dbl_3 tension $=0.1$ smooth $=20$ theta $=2.75$ scalex $=0.25$

Setting region depend on new pixel size

g.region vect=elevpoints rows $=16$ cols $=16$

Converting pixels to point data

r.to.vect input=elev_GPS output=centroit_points

feature $=$ point
Again setting resolution 1 meter

g.region vect $=$ elevpoints rows $=269$ cols $=275$

Creating elevation model in tension 40 smoothing 0 and without anisotropy angle and scale from last points

v.surf.rst input=centroit_points elev=elev_map zcolumn=value 